\title{
МЕТОД ДЕТЕРМИНИРОВАНИЯ СТРУКТУРЫ ИННОВАЦИОННЫХ ЭКОСИСТЕМ ВЫСОКОТЕХНОЛОГИЧНОГО СЕКТОРА
}

\author{
(C) 2021 Стародубов Денис Олегович
}

В настоящей публикации автором представлен развитый (по отношению к «индикативному», «сетевому», «ценностному» подходам) метод детерминирования структуры инновационных экосистем высокотехнологичного сектора. Сформулированы принципы и раскрыты итерации метода, позволяющего визуализировать инновационную экосистему, ее входы-выходы и оценить внутренние и внешние экономические потоки на примере телекоммуникационной индустрии. Метод направлен на совершенствование организационно-экономических параметров функционирующих и инициацию новых инновационных экосистем высокотехнологичного сектора.

Ключевые слова: инновации, экосистема, высокие технологии.

Организационная трансформация высокотехнологичного сектора обусловлена появлением новых механизмов инновационной кооперации субъектов (Макаров [1]). Наиболее обсуждаемым в научной среде является механизм «инновационных экосистем», феномен которого описывают (Moore [2]) по аналогии с биологическими экосистемами: «самоорганизация, взаимозависимость и взаимодополнение» (в контекстной проекции) экономических субъектов в инновационном цикле. Ключевой вопрос научной дискуссии об инновационных экосистемах - определение оптимальной структуры (состава субъектов и их взаимодействия), что позволяет тиражировать успешный опыт эффективных альянсов высокотехнологичного сектора. Именно поэтому наблюдается значительное количество публикаций, посвященных теоретическому поиску методов и подходов к картированию структуры экосистем.

В настоящее время сложилось 3 направления, подхода к детерминированию состава субъектов и их взаимосвязей в инновационных экосистемах. «Индикативный» (условные обозначения автором подходов, объединенных общим принципом) построен на количественной оценке инновационных «выходов» экосистемы (Huang и др. [3]), отражает агрегированный (средний) уровень инновационных показателей группы предприятий, входящих в экосистему. Его преимуществом является количественная оценка результативности и (или) эффективности кооперации, а ограничением отсутствие обоснованной платформы индикаторов, отражающих организационную специфику экосистемы как механизма. «Сетевой» (Nthubu [4]) построен на картировании взаимосвязи субъектов через контракты, бенефициаров или коммуникации, что создает преимущество в визуализации структуры, взаимосвязей субъектов. Но его применение ограничено отсутствием практической возможности получения информации о контрактах и (или) коммуникациях субъектов экосистемы. «Ценностный» (Talmara и др. [5]) peaлизуется как поиск «потребительской ценности» инновации при объединении ресурсов субъектов и позволяет (преимущество) выявить факторы взаимозависимости и взаимодополняемости в инновационном цикле. Но его ограничением является невозможность количественной оценки экономических «входов» и «выходов» экосистемы. Вместе с тем, все 3 подхода имеют и общее ограничение - отсутствует методическая определенность в выделении состава субъектов экосистем, не формулируются критерии (признаки) принадлежности.

С учетом обозначенных преимуществ и недостатков сложившихся подходов автором предложен развитый метод определения структуры, состава и взаимосвязи субъектов инновационных экосистем высокотехнологичного сектора, построенный на следующих теоретических посылах:

1. Признаком субъекта инновационной экосистемы определяется принадлежность вертикальной цепочке формирования добавленной стоимости технологической инновации, что отражает принцип «взаимозависимости и взаимодополняемости»;

2. Устойчивость инновационных экосистем определяется включенностью лидеров отраслей с различными активами, ключевыми для 
реализации инновационного цикла;

3. Структура отражается картированием (относительная позиция в экосистеме) и количественной оценкой, то есть визуализацией состава, взаимосвязей и их экономических пропорций.

Ключевым отличием и преимуществом авторского решения является введение критерия принадлежности субъектов инновационной экосистеме: включенность в вертикальную цепь формирования добавленной стоимости в инновационном цикле. Справедливости ради нужно отметить, что ранее аналогичные посылы формулировались («...моделируем инновационную экосистему как сеть фирм, связанных технологической зависимостью» (Jianxi [6]), но не были оформлены как критерий принадлежности, не воплощены в метод определения структуры. Данный критерий снимает общее для всех ранее сформированных подходов ограничение через «жесткий» критерий выявления границ экономических субъектов экосистемы. Основываясь на данном принципе, автор построил 5-и итерационный алгоритм реализации метода, графически представленный на рисунке 1 и раскры- тый в дальнейшем контексте публикации.

Итерация 1. Для инновационных экосистем высокотехнологичного сектора характерны «технологические инновации», то есть связанные «продуктовые» и «процессные» нововведения. Соответственно, в рамках итерации выделяют «продукт» (услугу) и «процесс» (технологию) конечного потребления физическими и(или) юридическими лицами, формируемые в инновационной кооперации экосистемы. Связанные продукты и услуги конечного потребления образуют «верхние узлы» (рис. 2) вертикальной цепи добавленной экосистемой стоимости. На примере телекоммуникационной индустрии (пример реализации метода в публикации): инновационный цикл воплощается в технически более совершенные услуги связи (например эволюция технологии сотовой связи - $1 \mathrm{G}, 2 \mathrm{G}, 3 \mathrm{G}, 4 \mathrm{G}, 5 \mathrm{G})$ и морально новое коммуникационное оборудование их поддерживающее. Соответственно, верхними узлами цепочки добавленной стоимости телекоммуникационной индустрии являются «услуги связи» и обеспечивающее их «коммуникационное оборудование» индивидуального (например, смартфоны) и промышленного (на-

\section{Итерации}

Содержание

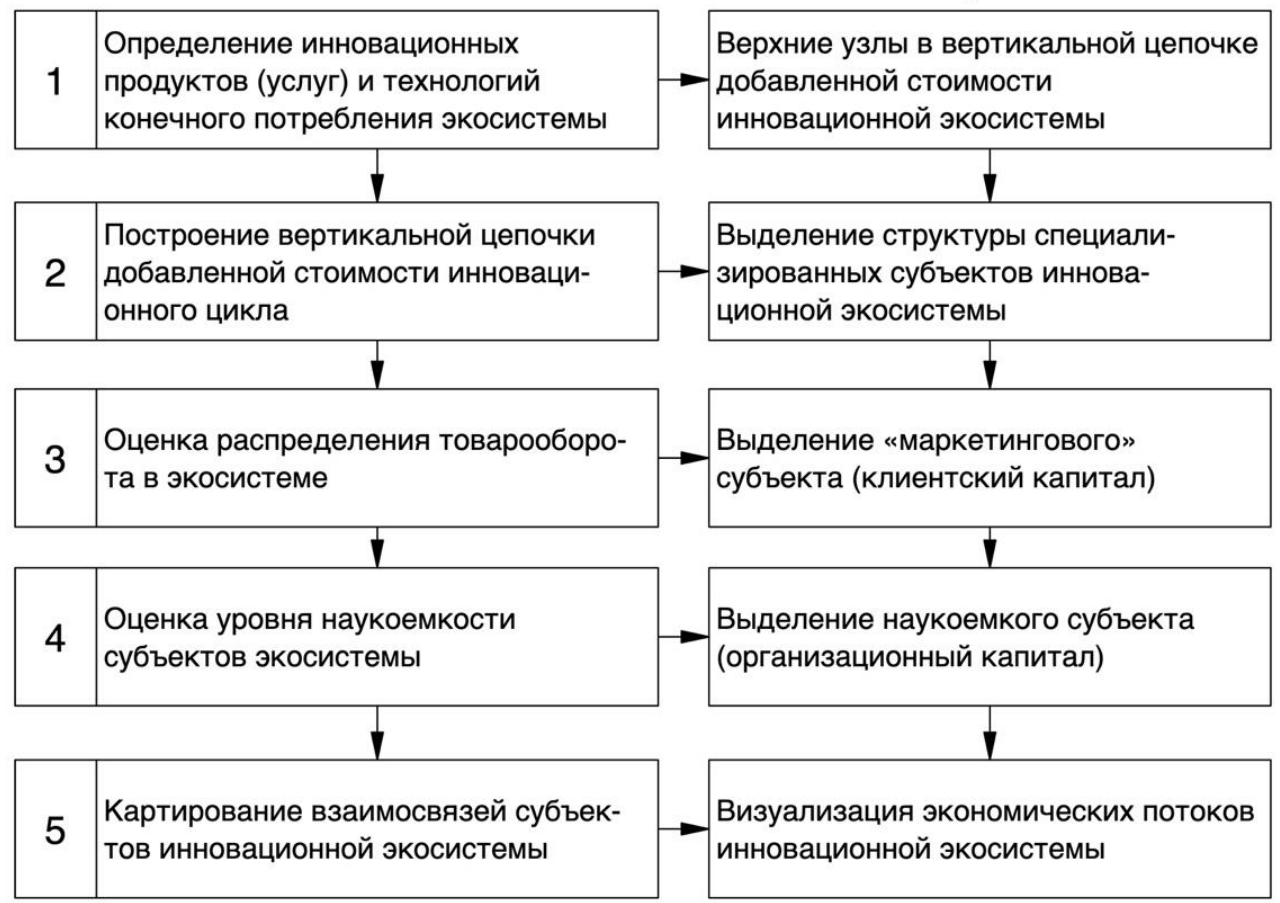

Puc. 1. Метод детерминирования структуры инновационной экосистемы высокотехнологичного сектора. Разработано автором. 
пример, трансляторы) назначения.

Итерация 2 как процесс построена на выявлении нисходящих узлов вертикальной цепочки добавленной стоимости, а как результат - определение состава специализированных субъектов, входящих в инновационную экосистему. Построение цепи основано на понимании движения ресурсов на этапах инновационного цикла (рис. 2). Критерием включения узла определяется ликвидность ресурса исключительно в рамках цепочки экосистемы. Так продукты «магнитные и оптические средства передачи информации» и услуги «строительства инженерных сооружений телекоммуникаций» ликвидны только в пределах телекоммуникационной индустрии (рис. 2) и не являются ресурсами для сторонних отраслей. А например, элементная база радиоэлектронной и приборостроительной промышленности (например, микросхемы) хотя и является ресурсом при производстве коммуникационного оборудования, но может быть реализована в широком спектре других отраслей (от автомобилестроения до геодезии). То есть, не выполняется принцип «взаимодополнения» и соответственно, несмотря на формальную принадлежность ресурсной цепи, данные узлы не включаются в экосистему.

Построенная вертикальная цепочка добавленной стоимости является основанием для выделения специализированных субъектов, включенных в инновационную экосистему. Алгоритмически: каждому узлу соответствует специализированный субъект, формирующий добавленную стоимость. Субъект относится к определённому виду деятельности (в исследовании использована интернациональная статистика, поэтому выбран классификатор NACE Rev. 2), то есть имеет отраслевую принадлежность. Выделенные субъекты (отрасли), образуют «индустрию», а в проекции на кооперацию в инновационном цикле - экосистему.

Фактически каждый субъект привязан к этапу инновационного цикла и имеет специализированные функции и активы, чем и выражает свою индивидуальную «ключевую компетенцию» (Hamel, Prahalad [7]). В этом и состоит

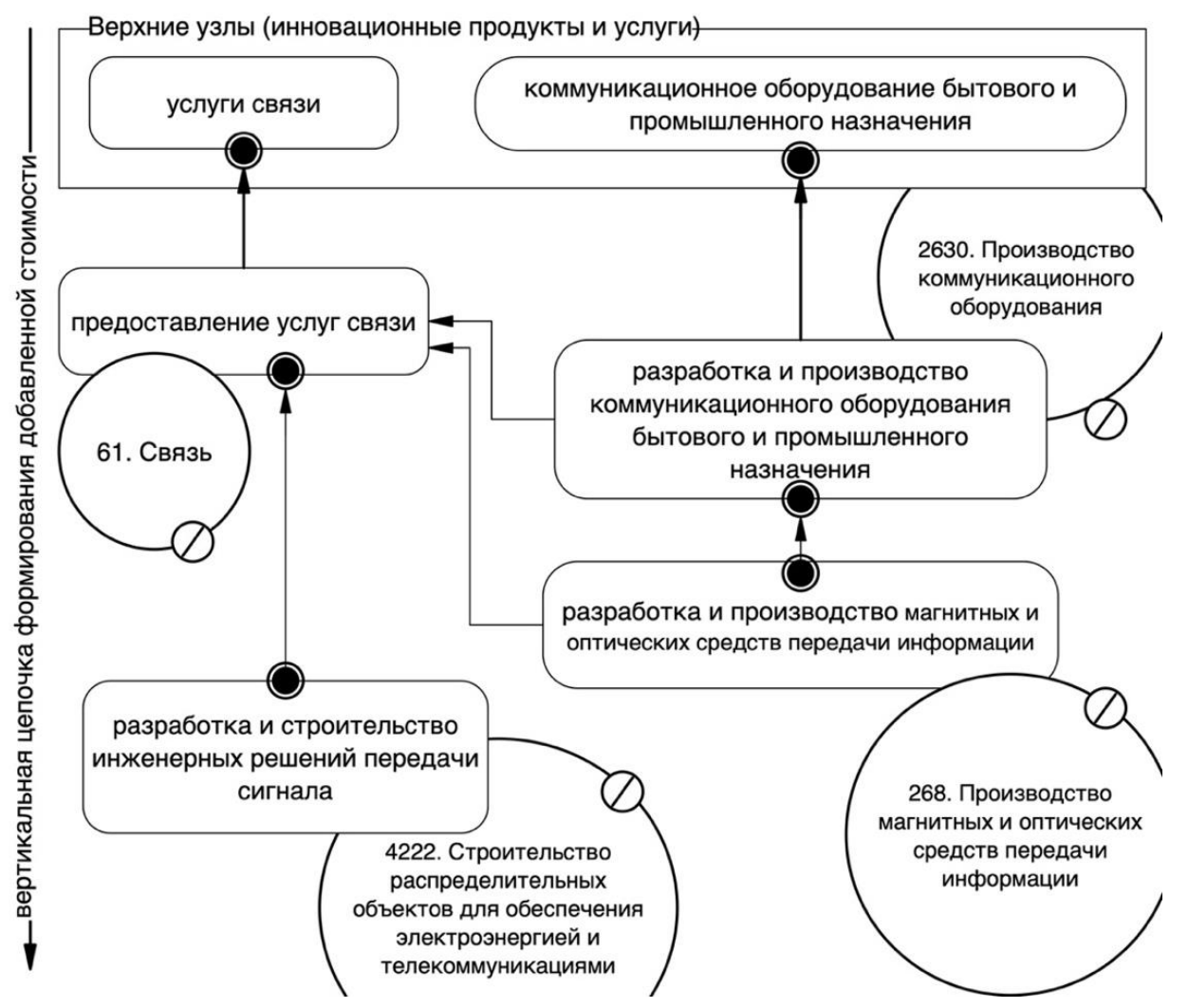

Puc. 2. Итерации 1-2: Узлы в вертикальной цепочке добавленной стоимости и специализированные субъекты $\oslash$ инновационной экосистемы (телекоммуникационная индустрия). 
принцип «взаимодополнения» экосистемы, отраженный как кооперация различных активов субъектов в инновационном цикле.

Применительно к телекоммуникационной индустрии выделено 4 субъекта инновационных экосистем, рис. 2. Автором сформирована выборка по базе данных Orbis из 157060 предприятий, относящихся к глобальной телекоммуникационной индустрии на 2020 год, что позволило оценить базовые пропорции экосистемы (результаты расчетов на рис. 3). Пропорции определяют ядро экосистемы, ее лидеров, формирующих долгосрочную устойчивость инновационного развития. Субъекты ядра - это поставщики услуг связи (80,01\% прибыли и $51 \%$ численности экосистемы) и разработчикипроизводители коммуникационного оборудования (18,35\% и 33\% соответственно).

Таким образом, итерация 2 позволила ограничить состав специализированных субъектов, определить их позицию в вертикальной цепочке формирования добавленной стоимости технологической инновации и выделить ядро высокотехнологичной инновационной экосистемы.

Итерация 3 направлена на поиск «маркетингового агента» экосистемы, носителя «клиентского» капитала, обеспечивающего коммерциализацию (этап цикла) технологической инновации. С позиции подхода к поиску автор исходит из положения, что маркетинговый агент консолидирует товарооборот экосистемы, вторично распределяя (рис. 3) экономические потоки по нисходящей цепочке формирования добавленной стоимости. Применительно к вы- борке 157060-и субъектов телекоммуникационной экосистемы автор построил распределение по отраслям и квартилям размерности предприятий (табл. 1), что позволило обнаружить маркетингового лидера - субъект предоставляющий услуги связи (71,15\% оборота экосистемы).

Важным результатом анализа распределения также следует считать концентрацию выручки крупных (70,99\% - 4-я квартиль оборот >1266 млрд. доллар США) поставщиков услуг связи. Это указывает на наличие глобальных маркетинговых лидеров в ядре инновационных экосистем, обеспечивающих ее устойчивость. А в теоретической плоскости подчеркивает «лидерский», вертикальный, иерархический принцип построения экосистем как механизма инновационной кооперации.

Целью итерации 4 является выделение наукоемкого лидера в ядре инновационных экосистем, генерирующего идею моральной новизны технологической инновации и реализующего цикл исследований и разработок. С позиции вклада в инновационный цикл наукоемкий лидер привносит организационный капитал (патенты, технологии, ноу-хау, другие объекты интеллектуальной собственности).

В этом контексте уместно обозначить авторскую позицию о взаимодействии субъектов «промышленность» и «научноисследовательские институты (организации)» в рамках инновационных экосистем. Несмотря на видимую «..очевидность принадлежности исследовательских организаций инновационной экосистеме» (Ander [8]), изученные автором кей-

Таблица 1. Распределение выручки и расходов на НИОКР в инновационной экосистеме высокотехнологичного сектора (на примере телекоммуникационной индустрии) в 2020 году по базовой выборке 157060 предприятий (база данных Orbis, расчеты автора).

\begin{tabular}{|c|c|c|c|c|c|}
\hline \multirow{2}{*}{$\begin{array}{l}\text { Отрасли (NACE Rev. 2) инновационной } \\
\text { экосистемы. Обозначение согласно рис. } 1 .\end{array}$} & \multicolumn{5}{|c|}{ Выручка (млрд. доллар США) по квартилям } \\
\hline & $<26$ & $26-255$ & $225-1266$ & $>1266$ & всего \\
\hline \multicolumn{6}{|c|}{ Распределение выручки в экосистеме по сегментам (квартилям) } \\
\hline 61. & $0,02 \%$ & $0,03 \%$ & $0,11 \%$ & $70,99 \%$ & $71,15 \%$ \\
\hline 2630. & $0,00 \%$ & $0,00 \%$ & $0,03 \%$ & $26,21 \%$ & $26,25 \%$ \\
\hline 268. & $0,00 \%$ & $0,00 \%$ & $0,00 \%$ & $0,06 \%$ & $0,06 \%$ \\
\hline 4222. & $0,00 \%$ & $0,01 \%$ & $0,08 \%$ & $2,46 \%$ & $2,55 \%$ \\
\hline Bce & $0,02 \%$ & $0,04 \%$ & $0,23 \%$ & $99,71 \%$ & $100,00 \%$ \\
\hline \multicolumn{6}{|c|}{ Распределение расходов на НИОКР в индустрии по сегментам (квартилям) } \\
\hline 61. & $0,01 \%$ & $0,00 \%$ & $0,01 \%$ & $13,57 \%$ & $13,59 \%$ \\
\hline 2630. & $0,00 \%$ & $0,00 \%$ & $0,04 \%$ & $86,36 \%$ & $86,40 \%$ \\
\hline 268. & $0,00 \%$ & $0,00 \%$ & $0,00 \%$ & $0,01 \%$ & $0,01 \%$ \\
\hline 4222. & $0,00 \%$ & $0,00 \%$ & $0,00 \%$ & $0,00 \%$ & $0,00 \%$ \\
\hline Bce & $0,01 \%$ & $0,00 \%$ & $0,05 \%$ & $99,94 \%$ & $100,00 \%$ \\
\hline
\end{tabular}




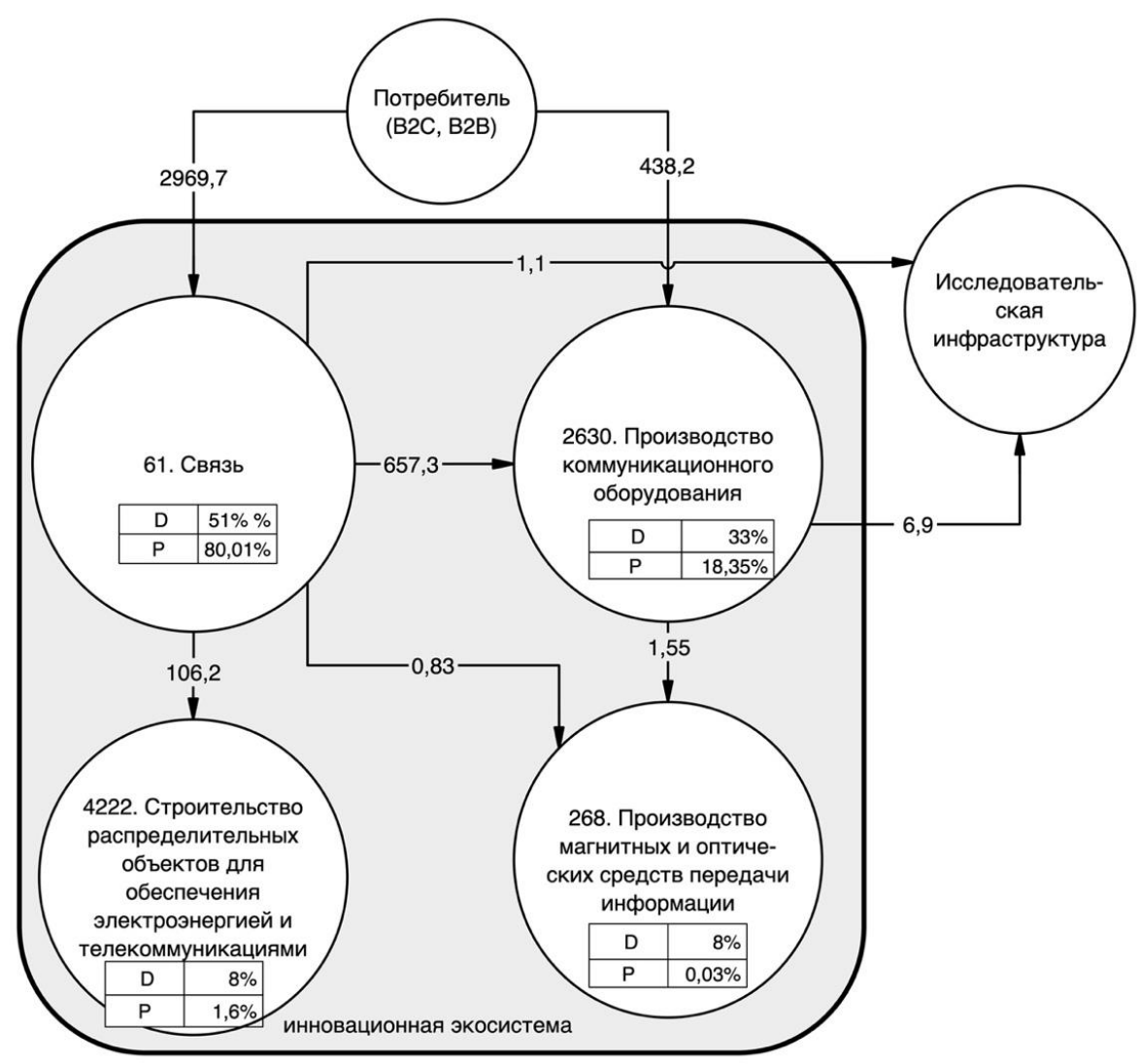

Puc. 3. Итерация 5: картирование инновационной экосистемы - телекоммуникационная индустрия. Обозн.: величины экономических взаимосвязей в млрд доллар США; стрелка заказчик $\rightarrow$ исполнитель контракта; D - доля субъектов в экосистеме в общей численности; Р - доля чистой прибыли экосистемы).

сы высокотехнологичного сектора обнаруживают иной контекст контрактного взаимодействия. Во-первых, 82\% (OECD, 2021 [9]) бюджета НИОКР высокотехнологичных промышленных предприятий адресовано внутренним исследовательским подразделениям и дочерним структурам. Во-вторых, с позиции экономических потоков расходы на НИОКР отражаются в бюджете высокотехнологичных промышленных предприятий, то есть в рамках инновационной экосистемы именно промышленность является собственником объектов интеллектуальной собственности (вне зависимости от структуры внешних подрядчиков НИОКР). В-третьих, авторское изучение кейсов высокотехнологичных экосистем показывает, что (узко) специализированные научные организации аффилированы промышленным концернам, являются либо дочерними структурами, либо аффилированы иным образом (оценка выполнена автором в рамках выборки через BvD индекс независимости капитала). А неаффилированные исследова- тельские организации, в том числе университетские научно-исследовательские подразделения, имеют широкую специализацию как с позиции лабораторной базы, так и с позиции научных компетенций ученых-исследователей. То есть, предложение независимых исследовательских организаций и университетских лабораторий является ликвидным за пределами экосистем, а субъекты не отвечают сформулированному принципу «взаимозависимости». В частности, в телекоммуникационной индустрии только 18\% (OECD [9]) исследований и разработок проводится за пределами экосистем, причем в очень узких единичных поисковых задачах, связанных с использованием узко специализированного лабораторного оборудования центров коллективного пользования.

Оценка распределения (табл. 1) расходов на НИОКР в телекоммуникационной экосистеме показывает, что 86,4\% от совокупных инвестиций в исследования сконцентрировано у производителей оборудования связи. Причем 86,36\% 
относится к крупным промышленным предприятиям - глобальным лидерам телекоммуникационной индустрии. Авторское исследование выборки показало, что 10 промышленных лидеров отрасли формируют 64\% мирового оборота высокотехнологичного коммуникационного оборудования (в совокупности потребительского и промышленного назначении).

Более того, 37\% материальных и 31\% нематериальных от совокупных активов отрасли консолидированы в первой десятке. Только одна компания Huawei Technologies имеет (по авторской оценке) 18\% мирового товарооборота коммуникационного оборудования.

Таким образом с методической точки зрения итерация метода (оценка распределения расходов на НИОКР) позволяет выделить наукоемкого лидера экосистемы, вносящего организационный капитал в инновационный цикл.

Завершающая метод 5-я итерация построена на визуализации (картировании) позиций субъектов инновационных экосистем и распределения внутренних и внешних (входы-выходы) экономических потоков, рис. 3. Методически итерация реализована в следующей последовательности. Во-первых, картируется, то есть определяется относительная позиция субъектов внутри экосистемы согласующаяся с принадлежностью цепочке формирования добавленной стоимости. Вторично определяется направленность и размерность внутренних и внешних экономических потоков инновационной экосистемы. И в заключение выносятся на карту пропорции субъектов по их численности и совокупной прибыли экосистемы.

Представленное на рис. 3 картирование телекоммуникационной экосистемы позволяет раскрыть аналитические возможности авторского метода. Во-первых, оценка пропорций обнаруживает лидерскую (иерархическую) модель организации высокотехнологичной (телекоммуникационной) экосистемы. То есть, экосистема самоорганизуется во взаимозависимую и взаимосвязанную структуру глобальных лидеров, формирующих ядро - поставщики услуг связи и производители телекоммуникационного оборудования. Во-вторых, ядро инновационных экосистем строится на взаимодополнении двух компонент интеллектуального капитала (клиентского и организационного), двух компетенций инновационного цикла (маркетинговой и научно-исследовательской). Объединение капитала различных по отраслевой принадлежности субъектов и рассматривается как инновационная кооперация высокотехнологичного сектора. В-третьих, объективно разделение и оценка размерности (объемный экономический показатель - оборот, интерпретируемый на рис. 3 через агрегированную годовую стоимость контрактов по видам деятельности) внутренних и внешних экономических потоков инновационной экосистемы: как в части реализации экосистемой инновационных услуг и продукции конченого потребления, так и входящий поток ресурсов.

Итак, представленный метод детерминирования структуры инновационных экосистем высокотехнологичного сектора демонстрирует преимущества по отношению к ранее сформированным подходам. Во-первых, он учитывает все достоинства «индикативного», «сетевого»и «ценностного» подходов: количественную оценку, визуализацию (картирование), понимание формирование потребительской ценности в кооперации. Во-вторых, снимает общее ограничение подходов, выраженное в неопределенности критериев отнесения субъектов к инновационной экосистеме. Предложенный автором критерий (принадлежность субъектов вертикальной цепочке формирования добавленной стоимости в инновационном цикле) апробирован в картировании глобальной телекоммуникационной экосистемы. В-третьих, представленный метод обладает широкими аналитическими возможностями для изучения кейсов эффективных инновационных экосистем, что позволяет перенести выявленные факторы успешности на существующие или создаваемые высокотехнологичные альянсы.

\section{Библиографический список}

1. Макаров B.B., Мохов А.С. Развитие сетевых форм взаимодействия в рамках национальных технологических инициатив как условие обеспечения конкурентоспособности российской экономики на глобальном рынке //Общество: политика, экономика, право. 2017. № 2. С. 30-33.

2. Moore, J.F. Predators and prey: A new ecology of competition. Harv. Bus. Rev. 1993, 71, 75-86. 
3. Huang, Xiaojing \& Ma, Lei \& Li, Rao \& Liu, Zheng. Determinants of Innovation Ecosystem in Underdeveloped Areas - Take Nanning High-Tech Zone in Western China as an Example. Journal of Open Innovation: Technology, Market, and Complexity, 2020. 6. 135.

4. Nthubu, Badziili \& Richards, \& Leon, Cruickshank. Disruptive Innovation Ecosystems: Reconceptualising Innovation Ecosystems. Conference: Disruptive Innovation Ecosystems: Reconceptualising Innovation EcosystemsAt: Academy for Design Innovation Management Conference, London, UK, 2019.

5. Talmar, Madis \& Walrave, Bob \& Podoynitsyna, Ksenia \& Holmström, Jan \& Romme, Georges. Mapping, analyzing and designing innovation ecosystems: The Ecosystem Pie Model. Long Range Planning, 53, 2020. 101850.

6. Jianxi. L. Architecture and Evolvability of Innovation Ecosystems. Technological Forecasting and Social Change, 2017. 136.

7. Hamel G., Prahalad C. K. Competing for the Future. Harvard Business School Press, April 1996.

8. Ander, R. Match your Innovation Strategy to your Innovation Ecosystem. Harv. Bus. Rev. 2006, 84, 83-98.

9. OECD Science, Technology and Innovation Outlook 2021: Times of Crisis and Opportunity, OECD Publishing, Paris, 2021. https://doi.org/10.1787/75f79015-en. 